International Journal of Engineering \& Technology, 7 (3) (2018) $1257-1262$
International Journal of Engineering \& Technology
SPC
Website: www.sciencepubco.com/index.php/IJET
doi: $10.14419 /$ ijet.v7i3.10099
Research paper

\title{
Dynamic modelling and analysis of 3D overhead gantry crane system
}

\author{
Khalil Azha Mohd Annuar ${ }^{1 *}$, Nik Azran Ab. Hadi, Mohamad Haniff Harun ${ }^{1}$, Mohd Firdaus Mohd Ab. Halim ${ }^{1}$, \\ Siti Nur Suhaila Mirin ${ }^{1}$, Arman Hadi Azahar ${ }^{1}$ \\ ${ }^{1}$ Center for Robotics and Industrial Automation (CeRIA), Department of Electrical Engineering Technology, Fakulti Teknologi \\ Keju-ruteraan (FTK), Universiti Teknikal Malaysia Melaka, Hang Tuah Jaya, 76100, Durian Tunggal, Melaka, Malaysia \\ *Corresponding author E-mail: khalilazha@utem.edu.my
}

\begin{abstract}
The overhead gantry crane systems are extensively used in harbours and factories for transportation of heavy loads. The crane speeding up, required for motion, always induces undesirable load swing. This writings present dynamic modelling of a 3D overhead gantry crane system based on closed-form equations of motion. By using the Lagrange technique, a 3D overhead gantry crane system nonlinear dynamic model is deriving. Then perform a linearization process to obtain a linear model dynamic system. Finally, simulation results systems responses of the derived nonlinear and linear model are presented showing the accuracy and performance of both model.
\end{abstract}

Keywords: 3D Overhead Crane System; Crane System; Lagrange Equation; Dynamic Model

\section{Introduction}

A 3D gantry crane system is used commonly to transport a large and heavy cargo in factories (Figure 1). But there is a number of serious problems occur in overhead cranes [1-4]. Example, crane acceleration or deceleration always induces undesirable load swing. In addition crane performance is also reduce or disturbed due to several factors including wind and rain. Other factors may also cause from a crane machinist may not effectively control a crane due to lack of care or lack of familiarity. Such problems decrease the work efficiency and in some cases cause damage to the loads and cause safety accidents.

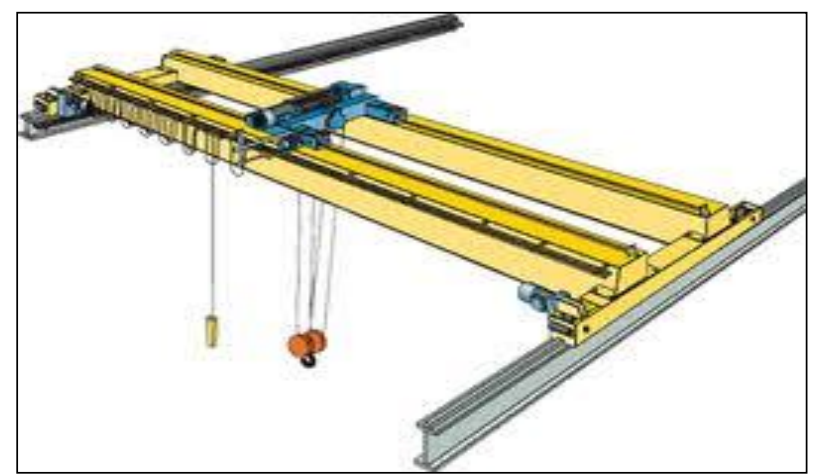

Fig. 1: Real 3D Overhead Crane System.

Therefore, research on the dynamic modelling of the framework crane system is crucial previous to any controller can be implemented to the system. A number of studies developed the dynamic representation and control design for 3D overhead gantry crane coordination. The effect of payload value on response of the system is study in [5-6]. The employment of dynamic crane model to de- termine an optimal speed that minimized load swing has been investigated and energy based nonlinear control for crane lifters has been reported in [7]. In these studies, complex system dynamic equations for a crane must be considered for the controller. Antiswinging control and motion planning for 3-D gantry and the study of the dynamic modelling of the other vibratory system incorporating payload is also has been reported [8].

While M. S. Omar et al. study on scheduling algorithm in order to find best performance in term of settling time and percentage of overshoot [9]. Another method scheduling optimization of RTGC by considering on moving distance, turning distance and practical operation rule [10].

This paper will study the nonlinear dynamic model equation of the system 3D overhead gantry crane system. Then perform a linearization process to obtain a linear model equation of the system. The Langrage principal is use to derive the dynamic model of the system. To analyse the accuracy and performance of both model, MATLAB software is used to simulate the system response of the models.

\section{System description}

The 3D overhead gantry crane system discuss in this work as shown in Figure 2 below. It consist [3] item in this system which is rail (r), cart (c) and payload (p). $\Theta$ and $\emptyset$ denote the swing angle of the rope, 1 is the length of rope, and $\mathrm{F}$ is the cart drive force. 


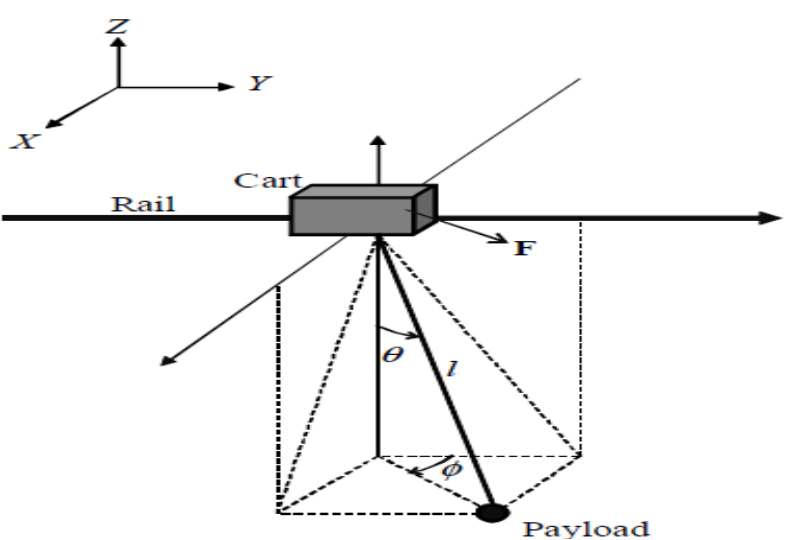

Fig. 2: Description of the 3D Overhead Gantry Crane.

For simplicity, the cart friction force is ignored. Also the cart and payload will be considered as point mass. The tension force that may cause the rope elongates is also ignored.

\section{3D Overhead gantry crane system mathe- matical model}

\subsection{Nonlinear mathematical model equation}

To derive the mathematical equation of the 3D overhead gantry crane system, the total energy associated with the crane system needs to be computed using the Lagrange method [11].

Based on Figure 2, the rail, cart and payload position vectors are given by,

rail position, $X_{r}=[x, 0,0]$

cart position, $\mathrm{X}_{\mathrm{c}}=[\mathrm{x}, \mathrm{y}, 0]$

payload position, $\mathrm{X}_{\mathrm{p}}=[\mathrm{x}+\mathrm{l} \sin \theta \sin \emptyset, \mathrm{y}+\mathrm{l} \sin \theta \cos \emptyset,-\mathrm{l} \cos \theta]$

Where $\mathrm{x}$ and $\mathrm{y}$ are the cart positions in $\mathrm{X}$ - and $\mathrm{Y}$-directions respectively.

Below given Lagrange equation formula is used,

$\frac{\partial}{\partial \mathrm{t}}\left(\frac{\mathrm{dl}}{\mathrm{dq} 1}\right)-\left(\frac{\mathrm{dl}}{\mathrm{dq}}\right)=\mathrm{Qi} \mathrm{i}=1,2,3,4$

Lagrangian function, $\mathrm{L}=\mathrm{T}-\mathrm{V}$

T: Kinetic energy

$\mathrm{V}$ : Potential energy

$\mathrm{Q}_{\mathrm{i}}$ : nonconservative generalized forces

Qi: independent generalized coordinate

$\mathrm{N}$ : total number of independent generalized coordinate

The state vector $\mathrm{q}$ and the control vector $\mathrm{F}$ are define as below,

State vector, $\mathrm{q}=[\mathrm{x}$ y $\theta \emptyset]$;

Control vector, $\mathrm{Q}_{\mathrm{T}}=\left[\mathrm{f}_{\mathrm{x}}, \mathrm{f}_{\mathrm{y}}, 0,0\right]^{\mathrm{T}}$

The total kinetic energy, $\mathrm{T}$ and potential energy, $\mathrm{V}$ of the whole system are given by

Total kinetic energy, $\mathrm{T}_{\text {total }}=\mathrm{T}_{\mathrm{r}}+\mathrm{T}_{\mathrm{c}}+\mathrm{T}_{\mathrm{p}}$

Kinetic energy for rail, $\mathrm{T}_{\mathrm{r}}=\frac{1}{2} \mathrm{mv}^{2}=\frac{1}{2} \mathrm{mr} \dot{\mathrm{x}}^{2}$

Kinetic energy for cart, $\mathrm{T}_{\mathrm{c}}=\frac{1}{2} \mathrm{mv}^{2}=\frac{1}{2} \mathrm{mrx}^{2}=\frac{1}{2} \mathrm{mcy}^{2}$
Kinetic energy for load, $\mathrm{T}_{\mathrm{p}}=\frac{1}{2} \mathrm{~m}_{\mathrm{p}} \dot{\mathrm{x}}^{2}+\frac{1}{2} \mathrm{~m}_{\mathrm{p}} \dot{\mathrm{y}}^{2}+$

$\mathrm{m}_{\mathrm{p}} \mathrm{l} \cos \theta \sin \emptyset \dot{\mathrm{x}} \dot{\theta}+\mathrm{m}_{\mathrm{p}} \mathrm{l} \sin \theta \cos \emptyset \dot{\varnothing} \dot{\mathrm{x}}+\mathrm{m}_{\mathrm{p}} \mathrm{l} \cos \theta \cos \emptyset \dot{\mathrm{y}} \dot{\theta}-$

$\mathrm{m}_{\mathrm{p}} \mathrm{l} \sin \theta \sin \emptyset \dot{\mathrm{y}} \dot{\emptyset}+\frac{1}{2}\left(\mathrm{~m}_{\mathrm{p}} \mathrm{l}^{2}+\mathrm{J}\right) \dot{\theta}^{2}+\frac{1}{2}\left(\mathrm{~m}_{\mathrm{p}} \mathrm{l}^{2} \sin ^{2} \theta+\mathrm{J}\right) \dot{\emptyset}^{2}$

Potential energy, $\mathrm{V}_{\text {total }}=\mathrm{Ve}+\mathrm{Vg}=$ Vpayload

$\mathrm{V}_{\text {total }}=-\mathrm{m}_{\mathrm{p}} \mathrm{gl} \cos \theta$

Where $\mathbf{J}$ denotes the moment of inertia of the payload and $\mathrm{g}$ is the gravity acceleration. Then the total energy and the total potential energy are obtained as

$\mathrm{L}=\mathrm{T}-\mathrm{V}$

$=\frac{1}{2}\left(m_{p}+m_{r}+m_{c}\right) \dot{x}^{2}+\frac{1}{2}\left(m_{p}+m_{c}\right) \dot{y}^{2}+$

$m_{p} l \cos \theta \sin \emptyset \dot{x} \dot{\theta}+m_{p} l \sin \theta \cos \emptyset \dot{\varnothing} \dot{x}$

$+m_{p} l \cos \emptyset \cos \emptyset \dot{y} \dot{\theta}-m_{p} l \sin \theta \sin \emptyset \dot{y} \dot{\varnothing}+\frac{1}{2}\left(m_{p} l^{2}+\right.$

$J) \dot{\theta}^{2}+\frac{1}{2}\left(m_{p} l^{2} \sin ^{2} \theta+J\right) \dot{\emptyset}^{2}-m_{p} g l \cos \theta$

When, $\mathrm{i}=1, \mathrm{q}=\mathrm{x}$

$\frac{\partial}{\partial t}\left(\frac{d l}{d \dot{x}}\right)-\frac{d l}{d x}=Q i$

$\frac{d l}{d x}=0$

$\frac{d l}{d \dot{x}}=\left(m_{p}+m_{r}+m_{c}\right) \dot{x}+m_{p} l \cos \theta \sin \emptyset \theta+m_{p} l \sin \theta \cos \emptyset \dot{\varnothing}$

$\frac{\partial}{\partial t}\left(\frac{d l}{d \dot{x}}\right)=\left(m_{p}+m_{r}+\right.$

$\left.m_{c}\right) \ddot{x}+m_{p} l \cos \theta \sin \emptyset \ddot{\theta}+m_{p} l \sin \theta \cos \emptyset \ddot{\emptyset}-m_{p} l \sin \theta \sin \emptyset \dot{\theta}^{2}$

$+m_{p} l \cos \theta \cos \emptyset \dot{\theta} \dot{\emptyset}+m_{p} l \cos \theta \cos \emptyset \dot{\theta} \emptyset-m_{p} l \sin \theta \sin \emptyset \dot{\emptyset}^{2}$

Substitute into Lagrange equation, $\frac{\partial}{\partial t}\left(\frac{d l}{d \dot{x}}\right)-\frac{d l}{d x}=Q i$

$=\left(m_{p}+m_{r}+m_{c}\right) \ddot{x}+m_{p} l \cos \theta \sin \emptyset \ddot{\theta}+m_{p} l \sin \theta \cos \emptyset \ddot{\varnothing}-$

$m_{p} l \sin \theta \sin \emptyset \dot{\theta}^{2}+m_{p} l \cos \theta \cos \emptyset \dot{\theta} \dot{\emptyset}+m_{p} l \cos \theta \cos \emptyset \dot{\theta} \dot{\emptyset}-$

$m_{p} l \sin \theta \sin \emptyset \dot{\emptyset}^{2}=f_{x}$

$\ddot{x}=$

$\underline{f_{x}-m_{p} l\left(\cos \theta \sin \varnothing \ddot{\theta}+\sin \theta \cos \varnothing \ddot{\varnothing}-\sin \theta \sin \varnothing \dot{\varnothing}^{2}+2 \cos \theta \cos \emptyset \dot{\theta} \dot{\varnothing}-\sin \theta \sin \varnothing \dot{\emptyset}^{2}\right.}$

When, $i=2, q=y$

$\frac{\partial L}{\partial y}=0$

$\frac{\partial L}{\partial \dot{y}}=\frac{1}{2}(2)\left(m_{p}+m_{c}\right) \dot{y}+m_{p} l \cos \theta \cos \emptyset \dot{\theta}-m_{p} l \sin \theta \sin \emptyset \dot{\varnothing}$

$\frac{\partial}{\partial t}\left(\frac{\partial L}{\partial \dot{y}}\right)=\left(m_{p}+m_{c}\right) \ddot{y}+m_{p} l \cos \theta \cos \emptyset \ddot{\theta}-$

$m_{p} l \sin \theta \sin \emptyset \ddot{\varnothing}-m_{p} l \sin \theta \cos \emptyset \dot{\theta}^{2}-m_{p} l \cos \theta \sin \emptyset \dot{\theta} \dot{\varnothing}-$ $m_{p} l \cos \theta \sin \emptyset \dot{\varnothing \dot{\theta}}-m_{p} l \sin \theta \cos \emptyset \dot{\theta}^{2}$

Substitute into Lagrange equation, $\frac{\partial y}{\partial x}\left(\frac{\partial y}{\partial x}\right)-\frac{\partial y}{\partial x}=Q_{i}$

$\left(m_{p}+m_{c}\right) \ddot{y}+m_{p} l \cos \theta \cos \emptyset \ddot{\theta}-m_{p} l \cos \theta \sin \emptyset \ddot{\varnothing}-$

$m_{p} l \sin \theta \cos \emptyset \dot{\theta}^{2}-m_{p} l \cos \theta \sin \emptyset \dot{\theta} \dot{\emptyset}-m_{p} l \cos \theta \sin \emptyset \dot{\emptyset} \dot{\theta}-$ $m_{p} l \sin \theta \cos \emptyset \dot{\theta}^{2}=F_{y}$

$y^{\prime \prime}=\left(F_{-} y-m \_p l(\cos \theta \cos \emptyset \theta \cdot-\cos \theta \sin \emptyset \emptyset “-\right.$

$\sin \theta \cos \emptyset \theta^{\wedge} 2-\cos \theta \sin \emptyset \theta^{*} \varnothing^{\circ}-\cos \theta \sin \emptyset \emptyset^{\circ} \theta^{*}-$

$\left.\left.\sin \theta \cos \emptyset \theta^{\wedge} 2\right)\right) /\left(\left(m_{-} p+m_{-} c\right)\right)$ 
When, $i=3, q=\theta$

$\frac{\partial y}{\partial x}=-m_{p} l \sin \theta \sin \emptyset \dot{x} \dot{\theta}+m_{p} l \cos \theta \cos \emptyset \dot{x} \dot{\varnothing}-$

$m_{p} l \sin \theta \cos \emptyset \dot{y} \dot{\theta}-m_{p} l \cos \theta \sin \emptyset \dot{y} \dot{\emptyset}+m_{p} l \sin \theta \cos \theta \dot{\emptyset}^{2}-$ $m_{p} y l \sin \theta$

$\frac{\partial L}{\partial \dot{\theta}}=m_{p} l \cos \theta \sin \emptyset \dot{x}+m_{p} l \cos \theta \cos \emptyset \dot{y}+m_{p} l^{2} \dot{\theta}$

$\frac{\partial}{\partial t}\left(\frac{\partial L}{\partial \dot{\theta}}\right)=m_{p} l \cos \theta \sin \emptyset \ddot{x}+m_{p} l \cos \theta \cos \emptyset \ddot{y}+m_{p} l^{2} \ddot{\theta}-$

$m_{p} l \sin \theta \sin \emptyset \dot{x} \dot{\theta}+m_{p} l \cos \theta \cos \emptyset \dot{x} \dot{\varnothing}-m_{p} l \sin \theta \cos \emptyset \dot{y} \dot{\theta}-$ $m_{p} l \cos \theta \sin \varnothing \dot{y} \dot{\varnothing}$

Substitute into Lagrange equation, $\frac{\partial}{\partial t}\left(\frac{\partial L}{\partial \dot{\theta}}\right)-\frac{\partial L}{\partial \theta}=Q_{i}$

$m_{p} l \cos \theta \sin \emptyset \ddot{x}+m_{p} l \cos \theta \cos \emptyset \ddot{y}+m_{p} l^{2} \ddot{\theta}-$

$m_{p} l \sin \theta \sin \emptyset \dot{x} \dot{\theta}+m_{p} l \cos \theta \cos \emptyset \dot{x} \dot{\theta}-m_{p} l \sin \theta \cos \emptyset \ddot{y} \dot{\theta}-$

$m_{p} l \cos \theta \sin \emptyset \dot{y} \dot{\varnothing}+m_{p} l \sin \theta \sin \emptyset \dot{x} \dot{\theta}-$

$m_{p} l \cos \theta \cos \emptyset \dot{x} \dot{\varnothing}+m_{p} l \sin \theta \cos \emptyset \dot{y} \dot{\theta}+$

$m_{p} l \cos \theta \sin \emptyset \dot{y} \dot{\varnothing}-m_{p} l^{2} \sin \theta \cos \theta \dot{\varnothing}^{2}+m_{p} l g l \sin \theta=0$

$m_{p} l \cos \theta \cos \emptyset \ddot{x}+m_{p} l \cos \theta \cos \emptyset \ddot{y}+\left(m_{p} l^{2}+J\right) \ddot{\theta}-$

$m_{p} l^{2} \sin \theta \cos \theta x \dot{\emptyset}^{2}+m_{p} g l \sin \theta=0$

$\ddot{\theta}=\frac{-m_{p} l\left(\cos \theta \cos \emptyset \ddot{x}+\cos \theta \cos \emptyset y-l \sin \theta \cos \ddot{\theta} x \dot{\emptyset}^{2}+g \sin \theta\right)}{\left(m_{p} l^{2}+J\right)}$

When, $i=4, q=\varnothing$

$\frac{\partial L}{\partial \emptyset}=m_{p} l \cos \theta \cos \emptyset \dot{x} \dot{\theta}-m_{p} l \sin \theta \sin \emptyset \dot{x} \dot{\varnothing}-$

$m_{p} l \cos \theta \sin \emptyset \dot{y} \dot{\theta}-m_{p} l \sin \theta \cos \emptyset \dot{y} \dot{\varnothing}$

$\frac{\partial L}{\partial \dot{\varnothing}}=m_{p} l \sin \theta \cos \emptyset \dot{x}-m_{p} l \sin \theta \sin \emptyset \dot{y}+\left(m_{p} l^{2} \sin ^{2} \theta+\right.$

$y) \dot{\varnothing}$

$\frac{\partial}{\partial t}\left(\frac{\partial L}{\partial \dot{\emptyset}}\right)=m_{p} l \sin \theta \cos \emptyset \ddot{x}-m_{p} l \sin \theta \sin \emptyset \ddot{y}+$

$\left(m_{p} l^{2} \sin ^{2} \theta+y\right) \ddot{\emptyset}+m_{p} l \cos \theta \cos \emptyset \dot{x} \dot{\theta}-$

$m_{p} l \sin \theta \sin \emptyset \dot{x} \dot{\emptyset}-m_{p} l \cos \theta \sin \emptyset \dot{y} \dot{\theta}-m_{p} l \sin \theta \cos \emptyset \dot{y} \dot{\emptyset}+$

$2 m_{p} l^{2} \cos \theta \sin \theta \dot{\varnothing} \dot{\theta}$

Substitute into Lagrange equation, $\frac{\partial}{\partial t}\left(\frac{\partial L}{\partial \dot{\emptyset}}\right)-\frac{\partial L}{\partial \emptyset}=Q_{i}$

$m_{p} l \sin \theta \cos \emptyset \ddot{x}-m_{p} l \sin \theta \sin \varnothing \ddot{y}+\left(m_{p} l^{2} \sin ^{2} \theta+J\right) \ddot{\varnothing}+$

$2 m_{p} l^{2} \cos \theta \sin \theta \dot{\varnothing} \dot{\theta}=0$

$\ddot{\emptyset}=\frac{-m_{p} l(\sin \theta \cos \emptyset \ddot{x}-\sin \theta \sin \emptyset \ddot{y}+2 l \cos \theta \sin \theta \dot{\varnothing} \dot{\theta})}{\left(m_{p} l^{2} \sin ^{2} \theta+y\right)}$

Comparison is done for the equation derive above (1), (2), (3) and (4) with the paper [1], where the equation derive is same.

\subsection{Linear mathematical model equation}

Based on the nonlinear equation in part A, first step is needed to determine the operating point of the system.

$$
\begin{aligned}
& x=\bar{x}, y=\bar{y}, \theta=\bar{\theta}, \phi=\bar{\phi} \\
& \dot{\bar{x}}=0, \dot{\bar{y}}=0, \dot{\bar{\theta}}=0, \dot{\bar{\phi}}=0 \\
& \ddot{\bar{x}}=0, \ddot{\bar{y}}=0, \ddot{\bar{\theta}}=0, \ddot{\bar{\phi}}=0
\end{aligned}
$$

Substitute to equation (1), (2), (3) and (4) then will get the operating point (equilibrium condition) of the system.

$(x=0, y=0, \theta=0, \phi=0)$ equilibium condition

Then to perform the linearization for all the nonlinear term as below:

Taylor series equation, $F(x, y, \theta, \emptyset)=F(\bar{\theta}, \bar{\phi})+\left.\frac{\partial F}{\partial \theta}\right|_{(\bar{\theta}, \bar{\phi})} \Delta \theta+$ $\left.\frac{\partial F}{\partial \phi}\right|_{\overline{(\theta, \bar{\phi})}} \Delta \emptyset$

$\cos \theta \sin \phi=\cos \bar{\theta} \sin \bar{\phi}+(-\sin \bar{\theta})(\sin \bar{\phi}) \Delta \theta+$ $\cos \bar{\theta} \cos \bar{\phi} \Delta \phi$

$\cos \theta \sin \phi=\cos 0 \sin 0+(-\sin 0)(\sin 0)+\cos 0 \cos 0 \Delta \phi$

$\cos \theta \sin \phi=0+0+\phi$

$\cos \theta \sin \phi=\phi$

$\sin \theta \sin \phi=\sin \bar{\theta} \sin \bar{\phi}+(\cos \bar{\theta}) \sin \bar{\phi} \Delta \theta+\sin \bar{\theta} \cos \bar{\phi} \Delta \phi$

$\sin \theta \sin \phi=\sin 0 \sin 0+\cos 0 \sin 0 \Delta \theta+\sin 0 \cos 0 \Delta \phi$

$\sin \theta \sin \phi=0$

$\cos \theta \cos \phi=\cos \bar{\theta} \cos \bar{\phi}+(-\sin \theta) \cos \phi \Delta \theta+$ $\cos \theta(-\sin \phi) \Delta \phi$

$\cos \theta \cos \phi=1$

$\sin \theta=\sin \bar{\theta}+\cos \bar{\theta} \Delta \theta$

$\sin \theta=0+1(\theta)$

$\sin \theta=\theta$

$\sin ^{2} \theta=\sin ^{2} \bar{\theta}+2 \sin \bar{\theta} \cos \bar{\theta} \Delta \theta$

$\sin ^{2} \theta=0$

To find the linear equation for this system by substitute all the term from equation (5) to (9) into the nonlinear equation (1) to (4).

Substitute into equation (1):

$\left(m_{p}+m_{v}+m_{c}\right) \ddot{x}+m_{p} l \phi \ddot{\theta}+m_{p} l \theta \ddot{\phi}+2 m_{p} l \dot{\theta} \dot{\phi}=F_{x}$

$\ddot{x}=\frac{F_{x}-m_{p} l(\phi \ddot{\theta}+\theta \ddot{\phi}+2 \ddot{\theta} \ddot{\phi})}{\left(m_{p}+m_{v}+m_{c}\right)}$

Substitute into equation (2):

$\left(m_{p}+m_{c}\right) \ddot{y}+m_{p} l \ddot{\theta}-2 m_{p} l \phi \ddot{\theta} \ddot{\phi}=F_{y}$

$\ddot{y}=\frac{F_{y}-m_{p} l(\ddot{\theta}-2 \phi \ddot{\theta} \ddot{\phi})}{\left(m_{p}+m_{c}\right)}$

Substitute into equation (3):

$m_{p} l \phi \ddot{x}+m_{p} l \ddot{y}+m_{p} l^{2} \ddot{\theta}-m_{p} \lg \theta=0$

$\ddot{\theta}=\frac{-m_{p} l(\phi \ddot{x}+\ddot{y}+g \theta)}{m_{p} l^{2}}$

Substitute into equation (4):

$m_{p} l \theta \ddot{x}+2 m_{p} l^{2} \theta \ddot{\phi} \ddot{\theta}+J \ddot{\phi}=0$ 
$\ddot{\phi}=\frac{-m_{p} l(\theta \ddot{x}+2 l \theta \ddot{\theta} \ddot{\phi})}{J}$

\section{Simulation results}

To investigate the characteristic of the model, the system has been built using MATLAB Simulink environment. Block diagram, consists of the system's block and input has been designed. Initially, the block diagram for nonlinear and linear in Figure 4 and 9 has been modelled:

A bang-bang signal of amplitude with value $1 \mathrm{~N}$ and $1 \mathrm{~s}$ width is used as input force has been applied at the cart of the gantry crane. A bang-bang force has a positive (acceleration) and negative (deceleration) period allowing the cart to, initially, accelerate and decelerate and eventually stop at the target location. System responses are verified by undertaking computer simulation using the fourth order Rungge kutta integration method for duration of $20 \mathrm{~s}$.

In order to investigate this simulation, the value of parameters has been considered.

Table 1: Parameter of the 3D Overhead Gantry Crane

\begin{tabular}{lll}
\hline Symbol & Parameter & Value \\
\hline $\mathrm{m}_{\mathrm{p}}$ & Payload mass & $1 \mathrm{~kg}$ \\
$\mathrm{~m}_{\mathrm{c}}$ & Cart mass & $1.06 \mathrm{~kg}$ \\
$\mathrm{~m}_{\mathrm{r}}$ & Rail mass & $6.4 \mathrm{~kg}$ \\
$\mathrm{l}$ & Rope length & $0.7 \mathrm{~m}$ \\
$\mathrm{~J}$ & Payload moment inertia & $0.005 \mathrm{~kg} \mathrm{~m}^{2}$ \\
$\mathrm{~g}$ & Gravity acceleration & $9.8 \mathrm{~m} \mathrm{~s}^{2}$ \\
\hline
\end{tabular}

\subsection{Nonlinear model equation simulation result}

From Figure 4 shows that the block diagram for nonlinear system $3 \mathrm{D}$ overhead crane system. Figure $5 \& 6$ show the response of the cart position in $\mathrm{X}$ and $\mathrm{Y}$ direction is almost same shape signal compare to paper [1]. It is noted that the average final position for both $\mathrm{X}$ and $\mathrm{Y}$ direction $0.14 \mathrm{~m}$ and $0.55 \mathrm{~m}$ with oscillation. Controller is needed to reduce the oscillation in this system.

For $\theta$ and $\emptyset$ graph will not compare to the paper [1] due to paper [1] only show the $\theta_{\mathrm{x}}$ and $\theta_{\mathrm{y}}$ angular.

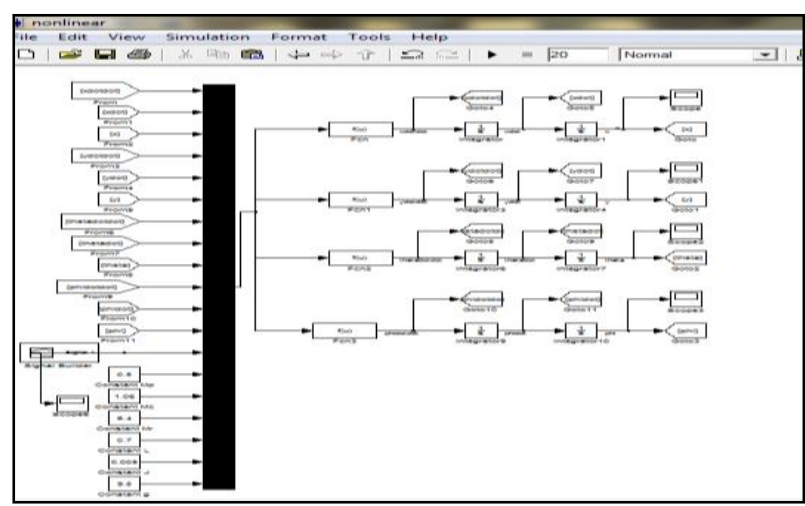

Fig.4: Nonlinear Block Diagram.

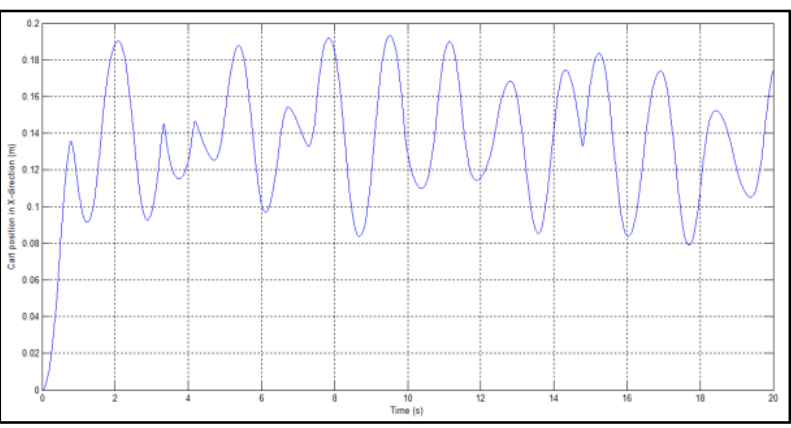

Fig. 5: Position of the Cart in X-Direction.

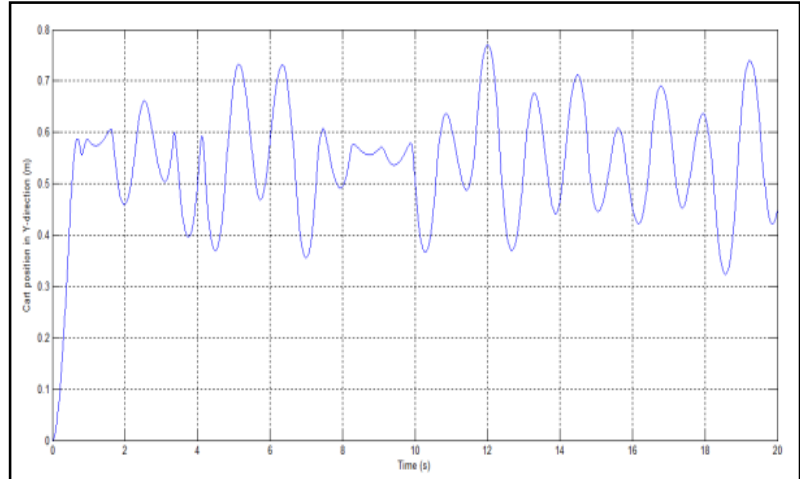

Fig. 6: Position of the Cart in X-Direction

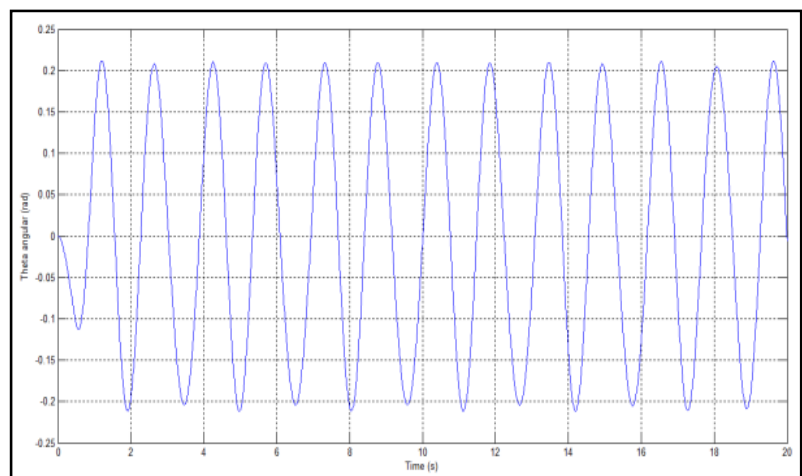

Fig. 7: Angle of the $\Theta$ Position

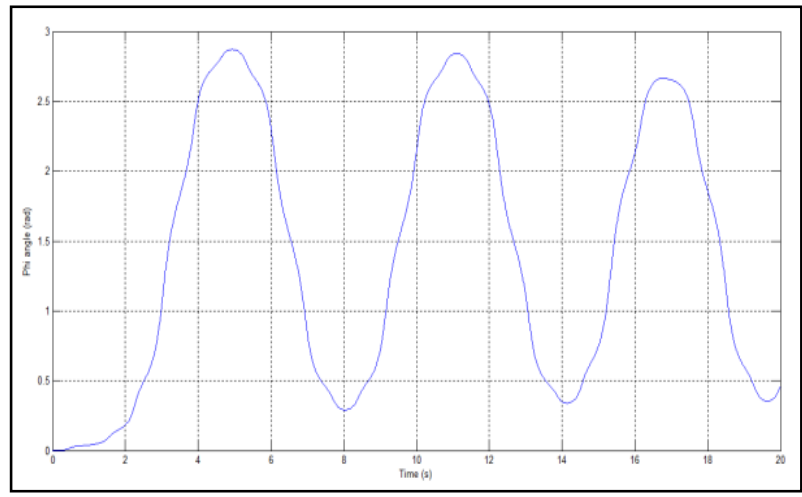

Fig. 8: Angle of the $\emptyset$

\subsection{Linear model equation simulation result}

Figure 9 shows the block diagram for linear model diagram. In linear model simulation it have limitation on sampling time only can simulate until $1.1 \mathrm{~s}$, there may be a singularity in the solution. From figure 10 to 13 it show the graph of linear model.

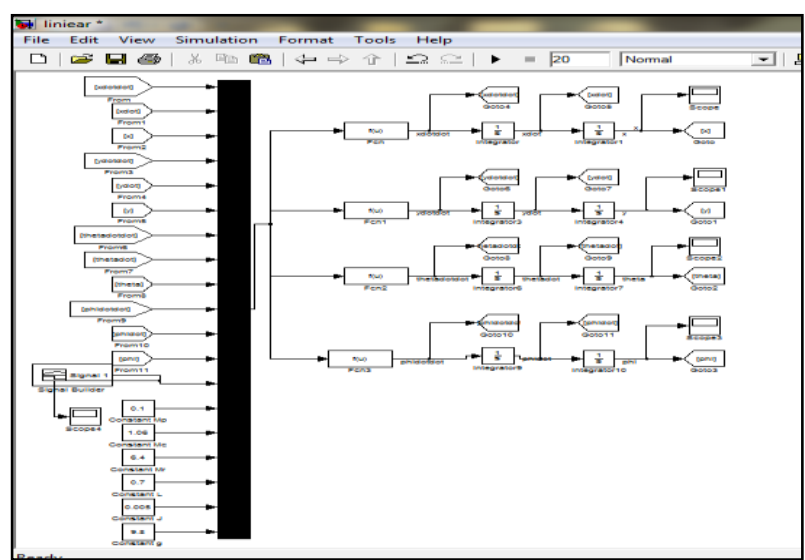

Fig.9: Linear Block Diagram. 


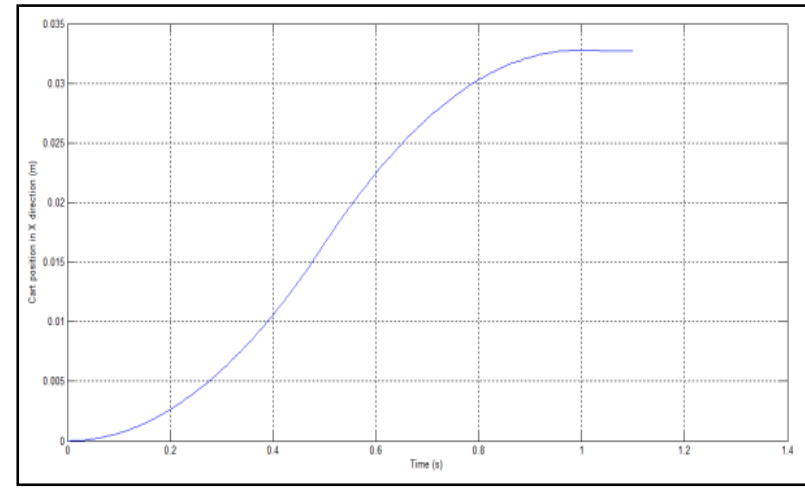

Fig. 10: Position of the Cart in X-Direction for Linear Model Equation

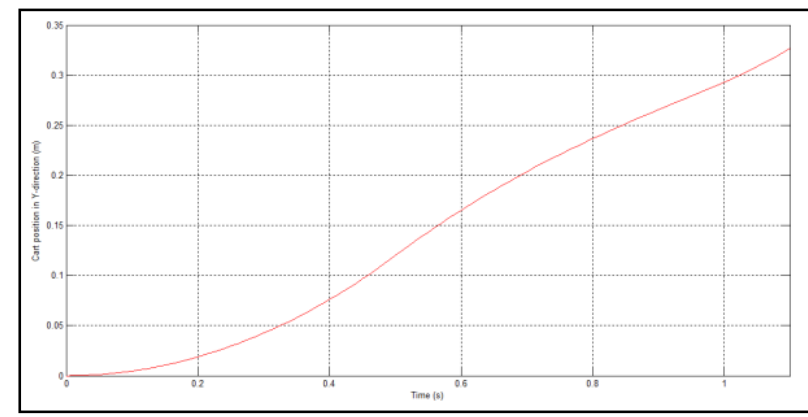

Fig. 11: Position of the Cart in Y-Direction for Linear Model Equation

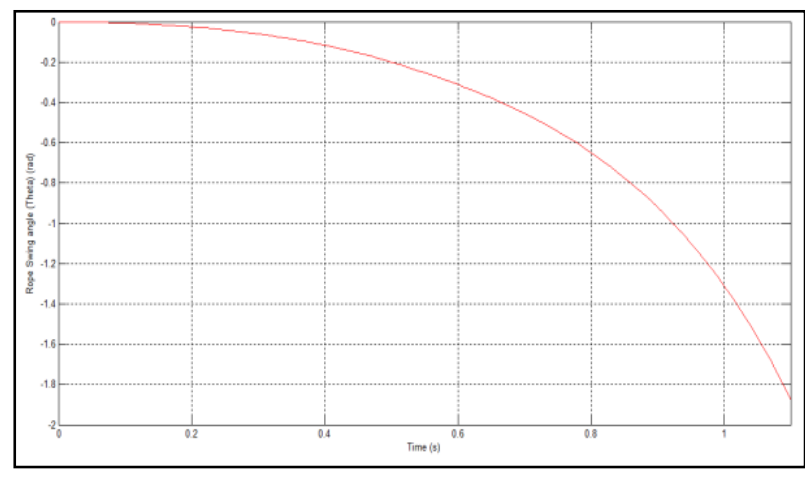

Fig. 12: Angle in $\Theta$ for Linear Model Equation.

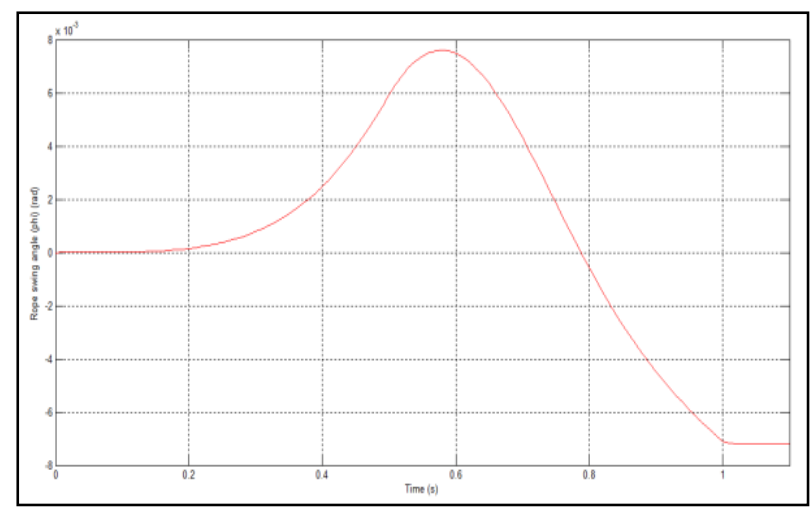

Fig. 13: Angle in $\emptyset$ for Linear Model Equation.

\section{Discussion}

To demonstrate the accuracy and performance of both models, the two of model equation is plot into same graph. The comparison is made only in duration 1.1s due to linear model equation limitation. Based on Figure 14, it shows that both plot is almost same, means the position value equation will give same value in linear and nonlinear equation. It shows the accuracy is good for $\mathrm{x}$ directions. For $\mathrm{Y}$ axis the gradient is for two models is almost same. By the way for $\theta$ and $\varnothing$ angular do not get same value for both graphs.

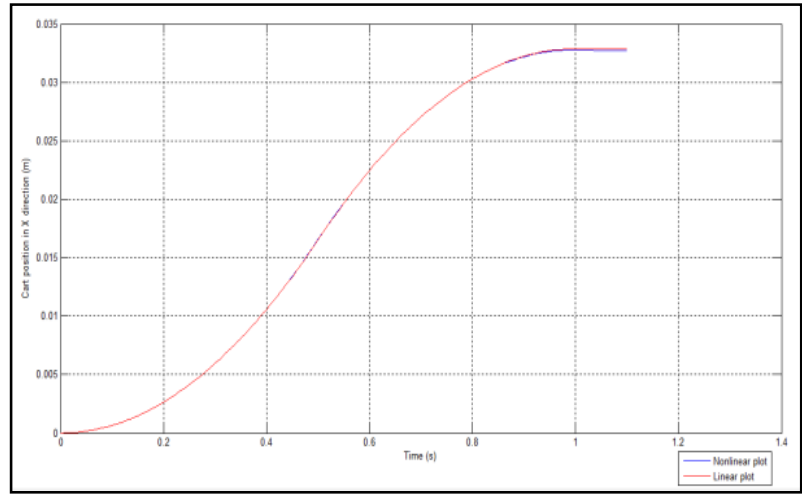

Fig. 14: Comparison between Nonlinear and Linear Model Equation (X Position).

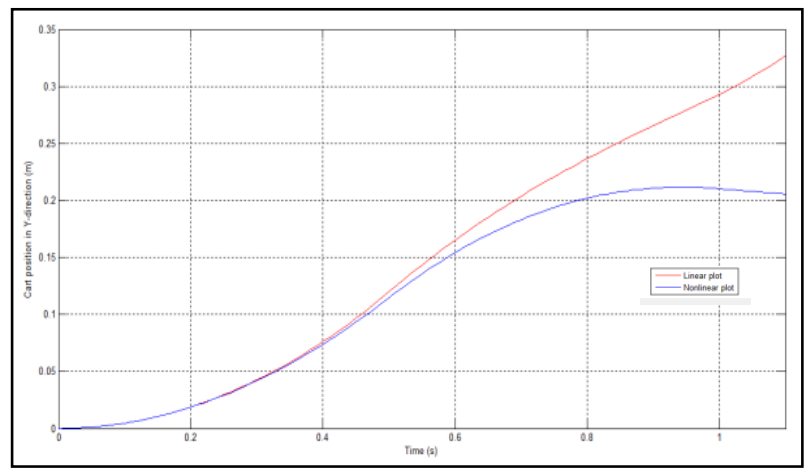

Fig. 15: Comparison between Nonlinear and Linear Model Equation (Y Position).

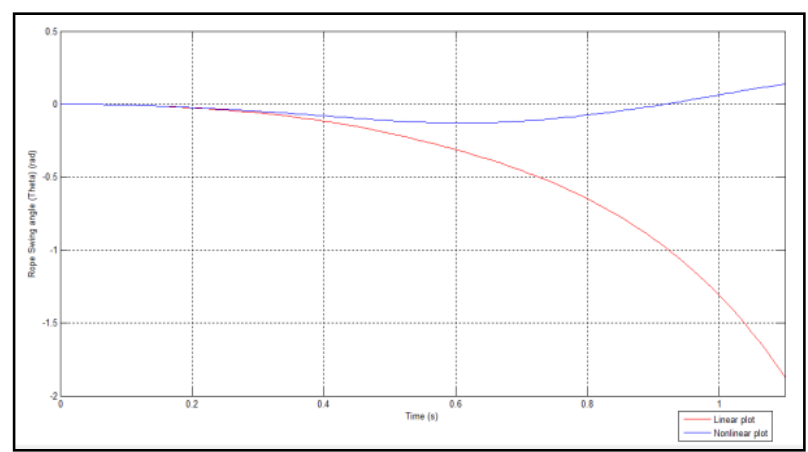

Fig. 16: Comparison between Nonlinear and Linear Model Equation $(\Theta$ Position)

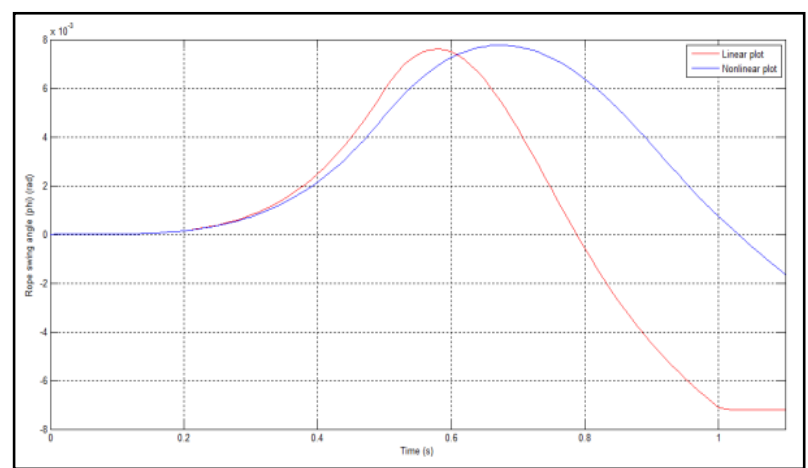

Fig. 17: Comparison between Nonlinear and Linear Model Equation ( $\varnothing$ Position)

\section{Conclusion}

The paper studies with dynamic model for 3D gantry crane system with nonlinear and linear model equation have been presented. The nonlinear model equation and graph shows that the result is almost same with the article [1]. The cart position and angular value response of gantry system has been obtained and analyzed in time 
domain. But linearization process to obtain linear equation where linear model graph is not as expected graph after compared to nonlinear model equation. Limitation problem in simulating linear model also will cause difficult to see the performance between nonlinear model and linear model equation.

Acknowledgement

The authors would like to thanks for the support given to this research by Ministry of Higher Education Malaysia and Universiti Teknikal Malaysia Melaka (UTeM) for support this under RAGS/1/2015/TK0/FTK/03/B00118 research grant.

\section{References}

[1] Leila Ranjbari, Amir H. Shirdel, M. Aslahi-Shahri, S. Anbari, A. Ebrahimi, M. Darvishi, M. Alizadeh, Rasoul Rahmani, M. Seyedmahmoudian, "Designing Precision Fuzzy Controller for Load Swing of An Overhead Crane" Neural Computing and Applications, Volume 26, Issue 7, pp. 1555-1560, 2015. https://doi.org/10.1007/s00521-015-1825-Z.

[2] Liyana Ramli, Z. Mohamed, Auwalu M. Abdullahi, H. I. Jaafar and Izzuddin M. Lazim, "Control strategies for crane systems: A comprehensive review” Mechanical Systems and Signal Processing, Volume 95, October 2017, Pages 1-23. https://doi.org/10.1016/j.ymssp.2017.03.015.

[3] Xianqing Wu, Xiongxiong He, "Enhanced Damping-Based AntiSwing Control Method for Underactuated Overhead Cranes" IET Control Theory \& Applications, Volume 9, Issue 12, pp. 1893-1900, 2015. https://doi.org/10.1049/iet-cta.2014.1353.

[4] Naif B. Almutairi and Mohamed Zribi, "Fuzzy Controllers for a Gantry Crane System with Experimental Verifications" Mathematical Problems in Engineering, Article ID 1965923, pp. 1-17, 2016. https://doi.org/10.1155/2016/1965923.

[5] R.M.T. Raja Ismail, M.A. Ahmad, M.S. Ramli and F.R.M. Rashidi, "Nonlinear Dynamic Modelling and Analysis of a 3D Overhead Gantry Crane System with Payload Variation”, 2009 Third UKSim European Symposium on Computer Modelling and Simulation, pp. 350-354, 2009.

[6] Anh-Huy Vo, Quoc-Toan Truong, Ha-Quang-Thinh Ngo and QuocChi Nguyen, "Nonlinear Tracking Control Of A 3-D Overhead Crane With Friction And Payload Compensations" Mechatronics, Electrical Power \& Vehicular Technology, Vol. 7, Issue 1, p27-34, 2016. https://doi.org/10.14203/j.mev.2016.v7.27-34.

[7] M.A. Karkoub and M. Zaribi., "Modelling and Energy Based Nonlinear Control of Crane Lifters", IEEE Proc. Control Theory Application, Vol. 149, No. 3, 2002, pp. 209-216. https://doi.org/10.1049/ip-cta:20020402.

[8] Dragan Antic, Zoran Jovanovic, Stanisa Peric et al, "Anti-Swing Fuzzy Controller Applied in a 3D Crane System," ETASR - Engineering, Technology \& Applied Science Research, Vol. 2, No. 2, 2012, 196-200.

[9] M.S. Omar, H.I. Jaafar, R. Ghazali, S.H. Mohamad, K.A.M. Annuar, "Investigation of Single Cart Gantry Crane System Performance using Scheduling Algorithm” International Journal of Soft Computing and Engineering (IJSCE), Volume 4, Issue 6, pp. 134-138, 2015.

[10] Mei Sha, Tao Zhang, Ying Lan, Xin Zhou, Tianbao Qin, Dayong Yu and Kai Chen, "Scheduling optimization of yard cranes with minimal energy consumption at container terminals" Computers \& Industrial Engineering, Volume 113, pp. 704-713, 2017. https://doi.org/10.1016/j.cie.2016.03.022.

[11] Hongjun Chen, Bingtuan GAO and Xiaohua Zhang, "Dynamic Modelling and Nonlinear Control of a 3D Crane," International Conference on Control and Automation (ICCA2005) June 27-29, Budapest, Hungary, 2005. 